\title{
Forecasting the Acquisition of University Spin-Outs: An RBF Neural Network Approach
}

\author{
Weiwei Liu, ${ }^{1,2}$ Zhile Yang, ${ }^{3}$ and Kexin $\mathrm{Bi}^{1,4}$ \\ ${ }^{1}$ School of Economics and Management, Harbin Engineering University, Harbin 150001, China \\ ${ }^{2}$ Management School, Queen's University Belfast, Belfast BT9 5EE, UK \\ ${ }^{3}$ Shenzhen Institute of Advanced Technology, Chinese Academy of Sciences, Shenzhen 518055, China \\ ${ }^{4}$ School of Management, Harbin University of Science and Technology, Harbin 150080, China
}

Correspondence should be addressed to Zhile Yang; zyang07@qub.ac.uk

Received 14 July 2017; Accepted 14 September 2017; Published 17 October 2017

Academic Editor: Jing $\mathrm{Na}$

Copyright (C) 2017 Weiwei Liu et al. This is an open access article distributed under the Creative Commons Attribution License, which permits unrestricted use, distribution, and reproduction in any medium, provided the original work is properly cited.

\begin{abstract}
University spin-outs (USOs), creating businesses from university intellectual property, are a relatively common phenomena. As a knowledge transfer channel, the spin-out business model is attracting extensive attention. In this paper, the impacts of six equities on the acquisition of USOs, including founders, university, banks, business angels, venture capitals, and other equity, are comprehensively analyzed based on theoretical and empirical studies. Firstly, the average distribution of spin-out equity at formation is calculated based on the sample data of 350 UK USOs. According to this distribution, a radial basis function (RBF) neural network (NN) model is employed to forecast the effects of each equity on the acquisition. To improve the classification accuracy, the novel set-membership method is adopted in the training process of the RBF NN. Furthermore, a simulation test is carried out to measure the effects of six equities on the acquisition of USOs. The simulation results show that the increase of university's equity has a negative effect on the acquisition of USOs, whereas the increase of remaining five equities has positive effects. Finally, three suggestions are provided to promote the development and growth of USOs.
\end{abstract}

\section{Introduction}

Universities have the functions of talents training and scientific research. Moreover, policymakers, on the other hand, have increasing concerns on what the role universities can play in economic development. Universities are requested to make contributions to their region's economic development by promoting effective university-industry relationships to exploit and commercialize research discoveries. Exploiting scientific and technological developments in universities is a main theme in economic and industrial policy [1]. However, universities may not be able to capture the full value of their technology due to the limitation of the licensing arrangement. Therefore, spinning out a company becomes a more direct involvement in the commercialization of new technology [2]. Accordingly, there is a shift in emphasis from exploiting university intellectual property by licensing to a focus on spin-out activities [3], and to stimulate spin-outs has become a significant issue for both universities and governments [4].

However, USOs are familiar with other small high-tech firms and of poor ability to debt financing and also depend highly on financing equity for their growth. Therefore, as a financing channel, equity distribution of USOs is attracting extensive attention. Radial basis function (RBF) neural network (NN) method is a novel and effective feedforward neural network with best approximation and global optimal performance. The training method is fast and easy and has been successfully applied in many fields with its unique information processing capability, especially in the financial, economic, and management fields [5-8]. This study incorporates RBF NN to investigate the impacts of equity distribution on the acquisition of USOs, aiming to deliver quantitative references for policymakers, founders, and financing providers to promote the development and growth of USOs. 
Three major contributions of this study are concluded as follows:

(1) It is the first attempt to adopt RBF NN method for forecasting the acquisition of USOs, which can resolve the problems that are intractable for traditional statistical method.

(2) To improve the classification accuracy, a novel setmembership method is adopted in the training process of the RBF NN.

(3) The simulation results confirm the effects of six equities on the acquisition of USOs and therefore provide quantitatively management decision references for policymakers, founders, universities, financial providers, and so forth.

The remainder of the paper is organized as follows. Section 2 provides an overview of previous studies on USOs and RBF NN, followed by Section 3 in which data and methodology are demonstrated. Section 4 presents the numerical results and discussions. Section 5 concludes the paper with the major findings from the methods and their policy implications.

\section{Literature Review}

2.1. University Spin-Outs (USOs). Lockett and Wright (2005) defined USOs as new ventures that are dependent upon licensing or assignment of an institution's intellectual property for initiation [9]. Moreover, USOs can also be defined as firms established by university academics aiming at commercializing ideas based on scientific discoveries and inventions $[10,11]$. For many years, various definitions of university spin-outs have emerged, and scholars generally agree that the spin-outs derive from technologies developed within a university and the individuals who pursue their commercialization including scientific researchers, students, and graduates (Benner and Tushman, 2003 [12]; Steffensen et al., 2000 [13]; Klofsten and Jones-Evans, 2000 [14]). As a knowledge transfer channel, spin-out strategy is a key issue. Siegel et al. (2003) [3] pointed to the shift in emphasis from exploiting university intellectual property through licensing to a focus on spin-out activities.

The literatures in terms of spin-outs generally identify two categories of finance funding and support in the process of spin-out creation (De Coster and Butler, 2005 [15]; Landry et al., 2007 [16]): business angels and venture capitalists. Several evidences show a limited role for bank finance in spin-out creation in both UK and US studies (Roberts, 1991 [17]; EC, 2000a, b [18, 19]). Lockett and Wright (2005) stated that, in permitted conditions, the university may own equity in the spin-out, and some universities state that they encourage academic entrepreneurship and demand a share of the spinout equity, and there is a positive relation between new venturing and the involvement of academic founders in the spin-out equity (Muscio et al., 2016) [20].

2.2. Radial Basis Function (RBF) Neural Network (NN). Nowadays, RBF method has been a well-adopted tool in the stock market forecast and the prediction of nonlinear system [21-23]. An increasingly popular and promising approach to solve option pricing models is the use of numerical methods based on RBF [24]. Chan and Hubbert (2014) [25] demonstrated how European and American option prices can be computed under the jump-diffusion model using the RBF interpolation scheme. An implementation of RBF method for solving Black-Scholes-type partial differential equations (PDEs) is proposed to price the swaptions in the absence of credit risk [26]. Erdal and Ekinci make a comparison of various artificial intelligence methods in the prediction of bank failures, including support vector machines (SVMs), RBF NN, and multilayer perceptrons (MLPs), in addition to subjecting the explanatory variables to principal component analysis (PCA) [27]. RBF is also used for the valuation, optimization, market, margin, and credit risk management of gas-fired power plants and associated tolling contracts [28]. A four-phase dynamic feedback RBF model is established for supply partner selection in agile supply chains (ASCs) [29]. Kapetanios and Blake (2010) [30] propose new tests for the martingale difference restriction based on RBF NN. In summary, it can be seen that RBF neural network is widely used and developed in the fields of economy, management, finance, social sciences, and so forth.

Though the RBF has seen numerous applications in economic and management modeling areas, according to the authors' knowledge, it has not been found to be of use and of significant potentials in USOs relevant researches.

\section{Data and Methodology}

3.1. Data. In an effort to enhance our understanding of this sector, a database of 1044 active USOs was compiled from individual university records and Internet searches and matched to a published list of UK university spinouts [31]. Telephone interviews were conducted with UK USOs and a final sample of 350 was achieved. And finally we obtained several parts of the data, including finance, incubation, support, and intellectual property. According to the characteristics of the collected data, traditional statistical analysis method could not solve the problem well; therefore, this study attempts to use RBF neural network to test the influences of equity distribution on the acquisition of USOs.

In our survey of UK USOs, the distribution of equity was measured in 2014 when the survey was not only conducted, but also traced back when the business was formed. At the point when USOs are formed, on average the founders own $56.51(\mathrm{SD}=30.92)$ percent of equity, and $24.73(\mathrm{SD}=23.36)$ percent belongs to the host university. Financial providers own a relatively small share of the overall equity at this stage of development, with venture capitals accounting for 7.71 (SD = 16.90) percent followed by business angels at $2.24(\mathrm{SD}=8.37)$ percent and, to a much less extent, banks at $0.45(\mathrm{SD}=4.53)$ percent and others at $8.36(\mathrm{SD}=21.11)$ percent, as shown in Figure 1.

3.2. RBF Neural Network. RBF NN is a three-layer forward network, including input-layer, hidden-layer and outputlayer, as shown in Figure 2. The performance of RBF NN 


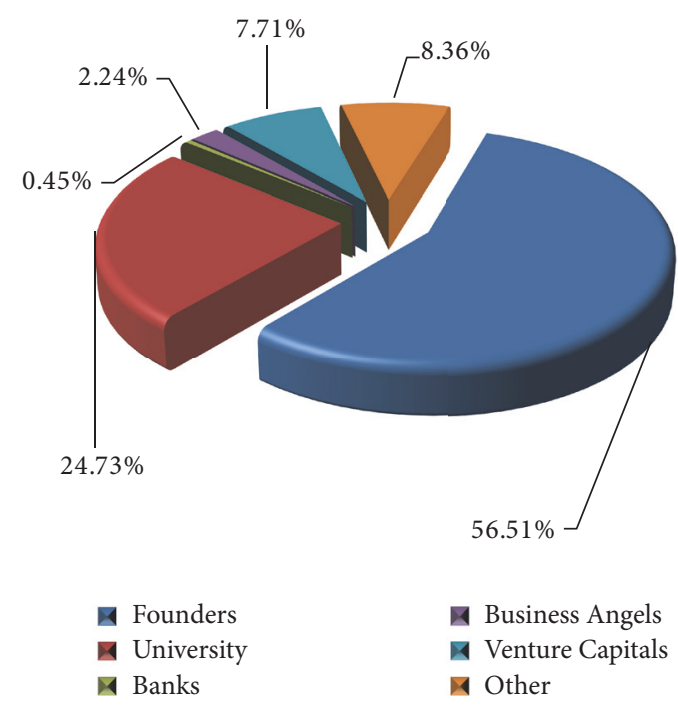

FIGURE 1: Average distribution of SPOs equity at formation (percentage).

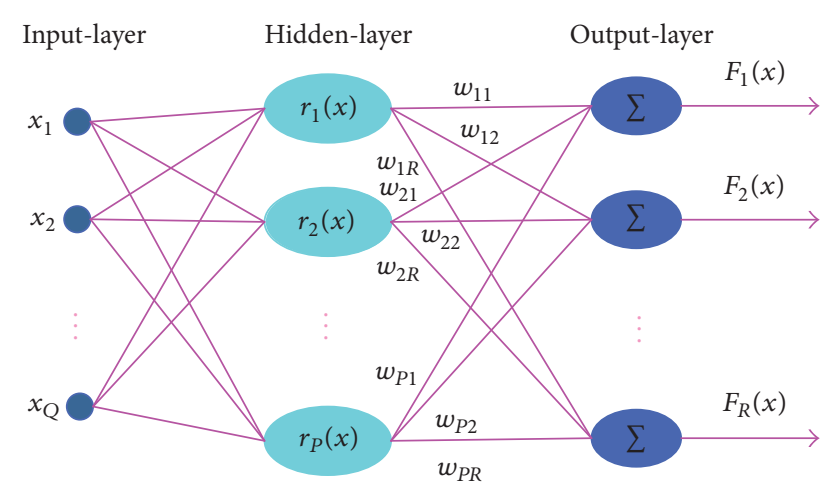

Figure 2: RBF NN model.

is determined by its topological parameters, such as the mean and variance of the RBF, the number of hidden nodes, and the weight between the hidden-layer and the outputlayer. Therefore, the training process of RBF NN focuses on adjusting the aforementioned factors so as to obtain precise model between inputs and outputs. Specifically, the location of RBF in the input space is influenced by its mean value, while the active zone is affected by its variance. The RBF or the neuron would be activated and produce large output if the input signal is close to its center. Otherwise, the output of RBF will tend to zero. Due to the limited response region of each RBF, the linear projection from hidden-layer to output-layer is needed to make the whole NN achieve specific function, such as classification and approximation [32].

The number of hidden nodes is a crucial factor in RBF $\mathrm{NN}$ : too little hidden nodes limit the learning ability, while a large number of hidden nodes lead to increasing training time and overfitting. According to the number of hidden nodes, the RBF NN is classified to generalized network (GN) model and regularized network (RN) model. By setting a restriction with prior knowledge, a smooth mapping function is obtained. The GN is suitable for classification. The input vectors in low-dimension space are transformed by the hidden-layer of NN, composed of RBF, achieving the projection to high-dimension space. Then, a linear-inseparable problem in low dimensional space becomes separable in high-dimension space.

Based on the study on the single-layer perceptron, a hyperplane described by linear equations exists if the modes in training sample space are linear-separable. This hyperplane would not exist when the training samples are nonlinearseparable. However, according to Cover theorem [33], the nonlinear-separable problem could be solved by nonlinear transformation. This nonlinear transformation is conducted by the hidden-layer of RBF NN.

The most commonly used radial base function is Gaussian function. If the hidden nodes number is $p$, the activation function of the $p$ th node is expressed in

$$
r_{p}(x)=\exp \left(-\frac{\left\|x-\mu_{p}\right\|_{2}^{2}}{2 \sigma_{p}^{2}}\right),
$$

where $\|\cdot\|_{2}$ denotes the norm of the vector, $\mu_{p}(p=$ $1,2, \ldots, P)$ denotes the center of the radial base function, and $\sigma_{p}$ denotes the variance of the Gaussian function. Suppose the numbers of neuron in input-layer and output-layer are $Q$ and $R$, respectively. Correspondingly, the input vector is $x=\left[x_{1}, x_{2}, \ldots, x_{\mathrm{Q}}\right]^{T}$ and the output vector is $y=$ $\left[y_{1}, y_{2}, \ldots, y_{R}\right]^{T}$. The activation functions of input-layer and output-layer are set to be identity function, and all the neuron thresholds are set to be zero. The weight between the hiddenlayer and the output-layer could be expressed as

$$
W=\left[\begin{array}{cccc}
w_{11} & w_{12} & \cdots & w_{1 R} \\
w_{21} & w_{22} & \cdots & w_{2 R} \\
\vdots & \vdots & \ddots & \vdots \\
w_{P 1} & w_{P 2} & \cdots & w_{P R}
\end{array}\right]
$$

Then the output of the $r$ th neuron in the output-layer is

$$
y_{r}=\sum_{r=1}^{R} w_{p r} r_{p}(x)=\sum_{r=1}^{R} w_{p r} \exp \left(-\frac{\left\|x-\mu_{p}\right\|_{2}^{2}}{2 \sigma_{p}^{2}}\right) .
$$

Once the topology of the RBF NN is determined, the accuracy of classification is depending on the coefficients matrix $W$, which could be optimized by the training process $[34,35]$

3.3. Set-Membership Training Method. Consider the following linear regression:

$$
y_{k}=G\left(x_{k}\right) \theta+\varsigma_{k}, \quad k=1,2, \ldots, n,
$$

where $y_{k} \in \mathfrak{R}^{n}$ is the output, $x_{k} \in \mathfrak{R}^{m}$ is the input, $\varsigma_{k}$ is the noise which is bounded by $\left\|c_{k}\right\|_{2} \leq r, \theta \in \mathfrak{R}^{q}$ is the parameter vector to be estimated, and $G\left(x_{k}\right)=\left[r_{1}\left(x_{k}\right), \ldots, r_{p}\left(x_{k}\right)\right] \epsilon$ $R^{n \times q}$. For the simplicity of the presentation, we use $G_{k}$ to denote $G\left(x_{k}\right)$ in the rest of the paper. 
Let $\theta_{k}$ be the estimate of $\theta$; the output of RBF neural network is

$$
\widehat{y}_{k}=G_{k} \theta_{k-1} .
$$

We define the training error as

$$
e_{k}=y_{k}-\widehat{y}_{k} \text {. }
$$

$\theta_{k}$ can be computed such that the loss function

$$
J_{k}=\sum_{i=1}^{k} \beta_{i} \frac{1}{r^{2}}\left\|e_{i}\right\|_{2}^{2}
$$

is minimized, where $\beta_{i}$ is the weighted factor to be determined.

The corresponding estimate is given by

$$
\theta_{k}=P_{k} \Phi_{k}
$$

with

$$
\begin{aligned}
P_{k}^{-1} & =\sum_{i=1}^{k} \beta_{i} \frac{1}{r^{2}} G_{i}^{T} G_{i} \\
\Phi_{k} & =\sum_{i=1}^{k} \beta_{i} \frac{1}{r^{2}} G_{i}^{T} y_{i} .
\end{aligned}
$$

We rewrite the above algorithm in the recursive form:

$$
\begin{aligned}
\theta_{k} & =\theta_{k-1}+\beta_{k} \frac{1}{r^{2}} P_{k} G_{k}^{T} e_{k}, \\
P_{k}^{-1} & =P_{k-1}^{-1}+\beta_{k} \frac{1}{r^{2}} G_{k}^{T} G_{k}
\end{aligned}
$$

which is also equivalent to the algorithm in the following Kalman filter form:

$$
\begin{aligned}
& \theta_{k}=\theta_{k-1}+\Gamma_{k} e_{k} \\
& \Gamma_{k}=\beta_{k} P_{k-1} G_{k}^{T}\left(\beta_{k} G_{k} P_{k-1} G_{k}^{T}+r^{2} I\right)^{-1} \\
& P_{k}=\left(I-\Gamma_{k} G_{k}\right) P_{k-1} .
\end{aligned}
$$

Until now, the design of the weighted factor $\beta_{k}$ is undetermined. It should be pointed out that the purpose of introducing the weighted factor $\beta_{k}$ is to measure the credibility of data $y_{k}$. If $y_{k}$ is more credible than $y_{k-1}$, the weighted factor $\beta_{k}$ should be larger than $\beta_{k-1}$. For instance, $\beta_{k}=0$ indicates that $y_{k}$ is not credible and should be neglected. For the design of the weighted factor $\beta_{k}$, the setmembership approach is employed. By reviewing the linear regression (4) and the boundedness property of the noise, $\theta$ is obviously in the set of the following form:

$$
\left\{\theta \in \mathfrak{R}^{q}: \frac{1}{r^{2}}\left(y_{i}-G_{i} \theta\right)^{T}\left(y_{i}-G_{i} \theta\right) \leq 1\right\} .
$$

For such $k$ sets of data, $\theta$ is then in the intersection of $k$ sets like (15), which can be expressed by

$\Omega(k)$

$$
=\bigcap_{i=1}^{k}\left\{\theta \in \mathfrak{R}^{q}: \frac{1}{r^{2}}\left(y_{i}-G_{i} \theta\right)^{T}\left(y_{i}-G_{i} \theta\right) \leq 1\right\} .
$$

Note that it is difficult to describe $\Omega(k)$ mathematically. However, it is convenient to design a set $\Theta(k)$ that contains $\Omega(k)$,

$$
\begin{aligned}
& \Theta(k)=\left\{\theta \in \Re^{q}: \sum_{i=1}^{k} \beta_{i} \frac{1}{r^{2}}\left(y_{i}-G_{i} \theta\right)^{T}\left(y_{i}-G_{i} \theta\right)\right. \\
& \left.\leq \sum_{i=1}^{k} \beta_{i}\right\} .
\end{aligned}
$$

Lemma 1. Given (12)-(14), the set $\Theta(k)$ given by (17) can be converted into the following ellipsoid expression:

$$
\Theta(k)=\left\{\theta \in \mathfrak{R}^{q}:\left(\theta-\theta_{k}\right)^{T} P_{k}^{-1}\left(\theta-\theta_{k}\right) \leq \sigma_{k}^{2}\right\} .
$$

Moreover, the recursive expression of $\sigma_{k}^{2}$ can be shown as below:

$$
\sigma_{k}^{2}=\sigma_{k-1}^{2}+\beta_{k}\left[1-e_{k}^{T}\left(\beta_{k} G_{k} P_{k-1} G_{k}^{T}+r^{2} I\right)^{-1} e_{k}\right] .
$$

Proof. Expanding (17) yields to

$$
\begin{aligned}
& \sum_{i=1}^{k} \beta_{i} \frac{1}{r^{2}} \theta^{T} G_{i}^{T} G_{i} \theta-\sum_{i=1}^{k} \beta_{i} \frac{1}{r^{2}} y_{i}^{T} G_{i} \theta-\sum_{i=1}^{k} \beta_{i} \frac{1}{r^{2}} \theta^{T} G_{i}^{T} y_{i} \\
& \quad \leq \sum_{i=1}^{k} \beta_{i}\left(1-\frac{1}{r^{2}} y_{i}^{T} y_{i}\right) .
\end{aligned}
$$

Substituting (9) into (20) leads to

$$
\theta^{T} P_{k}^{-1} \theta-\Phi_{k}^{T} \theta-\theta^{T} \Phi_{k} \leq \sum_{i=1}^{k} \beta_{i}\left(1-\frac{1}{r^{2}} y_{i}^{T} y_{i}\right) .
$$

Adding $\theta_{k}^{T} P_{k}^{-1} \theta_{k}$ to both sides of (21) leads to

$$
\begin{gathered}
\theta^{T} P_{k}^{-1} \theta-\Phi_{k}^{T} \theta-\theta^{T} \Phi_{k}+\theta_{k}^{T} P_{k}^{-1} \theta_{k} \\
\leq \sum_{i=1}^{k} \beta_{i}\left(1-\frac{1}{r^{2}} y_{i}^{T} y_{i}\right)+\theta_{k}^{T} P_{k}^{-1} \theta_{k} .
\end{gathered}
$$

Consider $\Phi_{k}=P_{k}^{-1} \theta_{k}$. The left side of (22) equals

$$
\begin{gathered}
\theta^{T} P_{k}^{-1} \theta-\Phi_{k}^{T} \theta-\theta^{T} \Phi_{k}+\theta_{k}^{T} P_{k}^{-1} \theta_{k} \\
=\left(\theta-\theta_{k}\right)^{T} P_{k}^{-1}\left(\theta-\theta_{k}\right) .
\end{gathered}
$$

Define the right side of (22) as the radius of the ellipsoid

$$
\sigma_{k}^{2}=\sum_{i=1}^{k} \beta_{i}\left(1-\frac{1}{r^{2}} y_{i}^{T} y_{i}\right)+\theta_{k}^{T} P_{k}^{-1} \theta_{k} .
$$

Thus, we can express $\Theta(k)$ by (15) in the ellipsoid form.

Moreover, from (24) the recursive form of $\sigma_{k}^{2}$ equals

$$
\begin{aligned}
\sigma_{k}^{2}= & \sigma_{k-1}^{2}+\beta_{k}\left(1-\frac{1}{r^{2}} y_{k}^{T} y_{k}\right)+\theta_{k}^{T} P_{k}^{-1} \theta_{k} \\
& -\theta_{k-1}^{T} P_{k-1}^{-1} \theta_{k-1} .
\end{aligned}
$$


Consider $\Phi_{k}=P_{k}^{-1} \theta_{k}$ and (10). The sum of the last two terms of (25) equals

$$
\begin{gathered}
\theta_{k}^{T} P_{k}^{-1} \theta_{k}-\theta_{k-1}^{T} P_{k-1}^{-1} \theta_{k-1}=\theta_{k}^{T} \Phi_{k}-\theta_{k-1}^{T} \Phi_{k-1} \\
\quad=\theta_{k-1}^{T} \Phi_{k}+\beta_{k} \frac{1}{r^{2}} e_{k}^{T} G_{k} P_{k} \Phi_{k}-\theta_{k-1}^{T} \Phi_{k-1} .
\end{gathered}
$$

Since $\Phi_{k}=\Phi_{k-1}+\beta_{k} \frac{1}{r^{2}} G_{k}^{T} y_{k}$, (26) further gives

$$
\begin{aligned}
\theta_{k}^{T} P_{k}^{-1} \theta_{k}-\theta_{k-1}^{T} P_{k-1}^{-1} \theta_{k-1} \\
=\beta_{k} \frac{1}{r^{2}} \theta_{k-1}^{T} G_{k}^{T} y_{k}+\beta_{k} \frac{1}{r^{2}} e_{k}^{T} G_{k} \theta_{k-1} \\
\quad+\beta_{k}^{2} \frac{1}{r^{4}} e_{k}^{T} G_{k} P_{k} G_{k}^{T} e_{k} .
\end{aligned}
$$

Substituting (27) into (25) results in

$$
\begin{aligned}
\sigma_{k}^{2} & =\sigma_{k-1}^{2}+\beta_{k}-\beta_{k} \frac{1}{r^{2}} e_{k}^{T} e_{k}+\beta_{k}^{2} \frac{1}{r^{4}} e_{k}^{T} G_{k} P_{k} G_{k}^{T} e_{k} \\
& =\sigma_{k-1}^{2}+\beta_{k}-\beta_{k} \frac{1}{r^{2}} e_{k}^{T}\left(I-G_{k} \Gamma_{k}\right) e_{k} \\
& =\sigma_{k-1}^{2}+\beta_{k}\left[1-e_{k}^{T}\left(\beta_{k-1} G_{k} P_{k-1} G_{k}^{T}+r^{2} I\right)^{-1} e_{k}\right] .
\end{aligned}
$$

That is the end of the proof.

On the basis of Lemma 1, we further transform (19) into that

$$
\begin{aligned}
\sigma_{k}^{2} \leq & \sigma_{k-1}^{2} \\
& +\beta_{k}\left[1-\lambda_{\min }\left\{\left(\beta_{k} G_{k} P_{k-1} G_{k}^{T}+r^{2} I\right)^{-1}\right\} e_{k}^{T} e_{k}\right] \\
= & \sigma_{k-1}^{2}+\beta_{k}\left[1-\frac{e_{k}^{T} e_{k}}{\lambda_{\max }\left\{\beta_{k} G_{k} P_{k-1} G_{k}^{T}+r^{2} I\right\}}\right] \\
= & \sigma_{k-1}^{2}+\beta_{k}\left[1-\frac{e_{k}^{T} e_{k}}{\left(\beta_{k}\left\|G_{k} P_{k-1} G_{k}^{T}\right\|_{2}+r^{2}\right)}\right] .
\end{aligned}
$$

Finally, we design $\beta_{k}$ as follows:

$$
\beta_{k}= \begin{cases}\frac{\left(\left\|e_{k}\right\|_{2}^{2}-r^{2}\right)}{\left\|G_{k} P_{k-1} G_{k}^{T}\right\|_{2}} & \left\|e_{k}\right\|_{2} \geq r \\ 0 & \text { otherwise. }\end{cases}
$$

Based on the above development, the main properties of the proposed training algorithm given by (12)-(14) are summarized in the following theorem.

Theorem 2. Consider the training algorithm given by (12)-(14), if we design the weighted factor $\beta_{k}$ according to (30), then the following statements are verified:

(1) If $\theta \in \Theta(0)$, then $\theta \in \Theta(k), \forall k$.

(2) $\left\|\theta-\theta_{k}\right\|_{2}$ is bounded and nonincreasing.

(3) $\lim _{k \rightarrow \infty}\left\|e_{k}\right\|_{2} \leq r$.
Proof. (1) Define $\widetilde{\theta}_{k}=\theta-\theta_{k}$ and choose the following Lyapunov function:

$$
V_{k}=\widetilde{\theta}_{k}^{T} P_{k}^{-1} \widetilde{\theta}_{k} .
$$

Substitution of (10) into the Lyapunov function leads to

$$
\begin{aligned}
& V_{k} \\
& =\left(\widetilde{\theta}_{k-1}-\beta_{k} \frac{1}{r^{2}} P_{k} G_{k}^{T} e_{k}\right)^{T} P_{k}^{-1}\left(\widetilde{\theta}_{k-1}-\beta_{k} \frac{1}{r^{2}} P_{k} G_{k}^{T} e_{k}\right) \\
& =\tilde{\theta}_{k-1}^{T} P_{k}^{-1} \widetilde{\theta}_{k-1}-\beta_{k} \frac{1}{r^{2}} e_{k}^{T} G_{k} \widetilde{\theta}_{k-1}-\beta_{k} \frac{1}{r^{2}} \widetilde{\theta}_{k-1}^{T} G_{k}^{T} e_{k} \\
& \quad+\beta_{k}^{2} \frac{1}{r^{4}} e_{k}^{T} G_{k} P_{k} G_{k}^{T} e_{k} .
\end{aligned}
$$

Recalling (11), (32) is expended to

$$
\begin{aligned}
V_{k}= & V_{k-1}+\beta_{k} \frac{1}{r^{2}} \widetilde{\theta}_{k-1}^{T} G_{k}^{T} G_{k} \widetilde{\theta}_{k-1}-\beta_{k} \frac{1}{r^{2}} e_{k}^{T} G_{k} \tilde{\theta}_{k-1} \\
& -\beta_{k} \frac{1}{r^{2}} \widetilde{\theta}_{k-1}^{T} G_{k}^{T} e_{k}+\beta_{k}^{2} \frac{1}{r^{4}} e_{k}^{T} G_{k} P_{k} G_{k}^{T} e_{k} .
\end{aligned}
$$

Since $\varsigma_{k}=e_{k}-G_{k} \widetilde{\theta}_{k-1}$, (33) is equivalent to

$$
\begin{aligned}
V= & V_{k-1}+\beta_{k} \frac{1}{r^{2}} \varsigma_{k}^{T} \varsigma_{k}-\beta_{k} \frac{1}{r^{2}} e_{k}^{T} e_{k} \\
& +\beta_{k}^{2} \frac{1}{r^{4}} e_{k}^{T} G_{k} P_{k} G_{k}^{T} e_{k} \\
= & V_{k-1}+\beta_{k} \frac{1}{r^{2}} \varsigma_{k}^{T} \varsigma_{k} \\
& -\beta_{k} \frac{1}{r^{2}} e_{k}^{T}\left(\mathbf{I}-\beta_{k} \frac{1}{r^{2}} G_{k} P_{k} G_{k}^{T}\right) e_{k} \\
= & V_{k-1}+\beta_{k} \frac{1}{r^{2}} \varsigma_{k}^{T} \varsigma_{k}-\beta_{k} \frac{1}{r^{2}} e_{k}^{T}\left(\mathbf{I}-G_{k} \Gamma_{k}\right) e_{k} .
\end{aligned}
$$

Substitution of the learning gain matrix presented by (13) yields to

$$
V_{k} \leq V_{k-1}+\beta_{k}\left[1-e_{k}^{T}\left(\beta_{k} G_{k} P_{k-1} G_{k}^{T}+r^{2} I\right)^{-1} e_{k}\right] .
$$

In observing (29), (35) equals

$$
V_{k} \leq V_{k-1}+\sigma_{k}^{2}-\sigma_{k-1}^{2}
$$

which can be rewritten as

$$
V_{k}-\sigma_{k}^{2} \leq V_{k-1}-\sigma_{k-1}^{2} .
$$

Then it follows that $V_{k-1} \leq \sigma_{k-1}^{2}$ deduces $V_{k} \leq \sigma_{k}^{2}$; namely, $\theta \in \Theta(k-1)$ deduces $\theta \in \Theta(k)$.

(2) From the proof of the statement (1), it follows that

$$
\begin{aligned}
V_{k} & \leq V_{k-1}+\beta_{k}\left[1-e_{k}^{T}\left(\beta_{k} G_{k} P_{k-1} G_{k}^{T}+r^{2} I\right)^{-1} e_{k}\right] \\
& \leq V_{k-1}+\beta_{k}\left[1-\frac{e_{k}^{T} e_{k}}{\left(\beta_{k}\left\|G_{k} P_{k-1} G_{k}^{T}\right\|_{2}+r^{2}\right)}\right] .
\end{aligned}
$$


Considering the design of $\beta_{k}$ given by (30), (38) further gives

$$
V_{k} \leq V_{k-1}
$$

which is equivalent to

$$
\widetilde{\theta}_{k}^{T} P_{k}^{-1} \widetilde{\theta}_{k} \leq \widetilde{\theta}_{k-1}^{T} P_{k-1}^{-1} \widetilde{\theta}_{k-1}
$$

Noting $P_{k}^{-1} \geq P_{k-1}^{-1}$, the following inequality holds

$$
\tilde{\theta}_{k}^{T} P_{k-1}^{-1} \tilde{\theta}_{k} \leq \tilde{\theta}_{k-1}^{T} P_{k-1}^{-1} \tilde{\theta}_{k-1}
$$

Thus $\widetilde{\theta}_{k}^{T} \widetilde{\theta}_{k} \leq \widetilde{\theta}_{k-1}^{T} \widetilde{\theta}_{k-1}$ is proved.

(3) The statement (3) will be verified for the following different cases.

Firstly, when $\lim _{k \rightarrow \infty} \sum_{i=1}^{k} \beta_{i}=\infty$, it results in $\lim _{k \rightarrow \infty} P_{k}^{-1}=\infty$ according to (6). Then we know that $\lim _{k \rightarrow \infty} \widetilde{\theta}_{k}^{T} \widetilde{\theta}_{k}=0$, due to $V_{k} \leq V_{k-1} \leq V_{0}<\infty$ which can be observed from the proof of statement (2). In this case, the statement (3) is straightforward.

Secondly, when $\lim _{k \rightarrow \infty} \sum_{i=1}^{k} \beta_{i}<\infty$, it leads to $\lim _{k \rightarrow \infty} \beta_{k}=0$. Thus, $\lim _{k \rightarrow \infty}\left\|e_{k}\right\|_{2} \leq r$ can be derived from (30).

That is the end of the entire proof.

Remark 3. The theoretical proof of this paper shows that, as the input data increases, the estimation values of the parameters will gradually approximate the true values of the parameters. This proof ensures the convergence of the estimation error.

\section{Results and Discussions}

We use the typical three-layer RBF neural network for the classification of 350 sets of data. In the RBF NN model, the input vector is six-dimensional and the output data is one-dimensional vector. We assume that there are 19 neural hidden nodes, and the width value of the Gaussian function for each neural net is initially chosen as 10 . The center point of the Gaussian function for the $i$ th neural net is chosen as $i \times[5,5,5,5,5,5]^{T}, i=1,2, \ldots, 19$. The weight value vector is trained by the proposed set-membership approach and the classification result can be shown in Figures 3 and 4 .

The vertical coordinates of Figures 3 or 4 denote the output values of the neural networks, which represent the possibilities of the companies being acquired. And the green diamonds in Figures 3 and 4 represent the acquired companies, and the red circles in Figure 3 represent the companies that have not been acquired.

The closer the output value is to 1 , the more likely the company is to be acquired. The closer the output value is to 1 , the more likely the company is to be acquired. The output value of neural network is continuous, but the acquisition is a Boolean variable. In order to achieve the purpose of the classification, we choose 0.5 as the dividing line (the blue line in Figures 3 and 4). The output value greater than 0.5 is on behalf of the company being acquired; otherwise the company will not be acquired. By comparing the neural

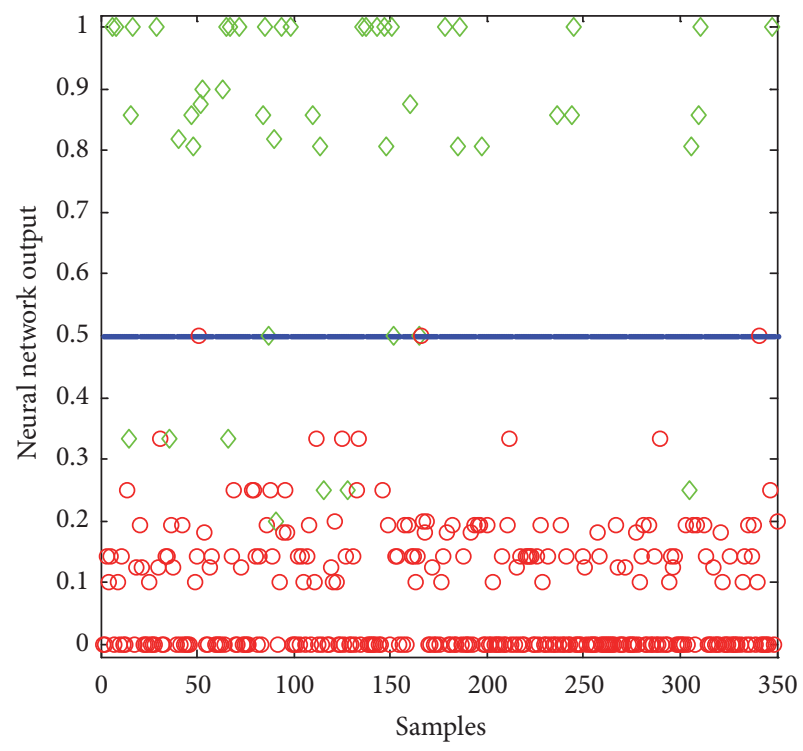

FIGURE 3: The RBF NN model training result (accuracy: 97.4\%).

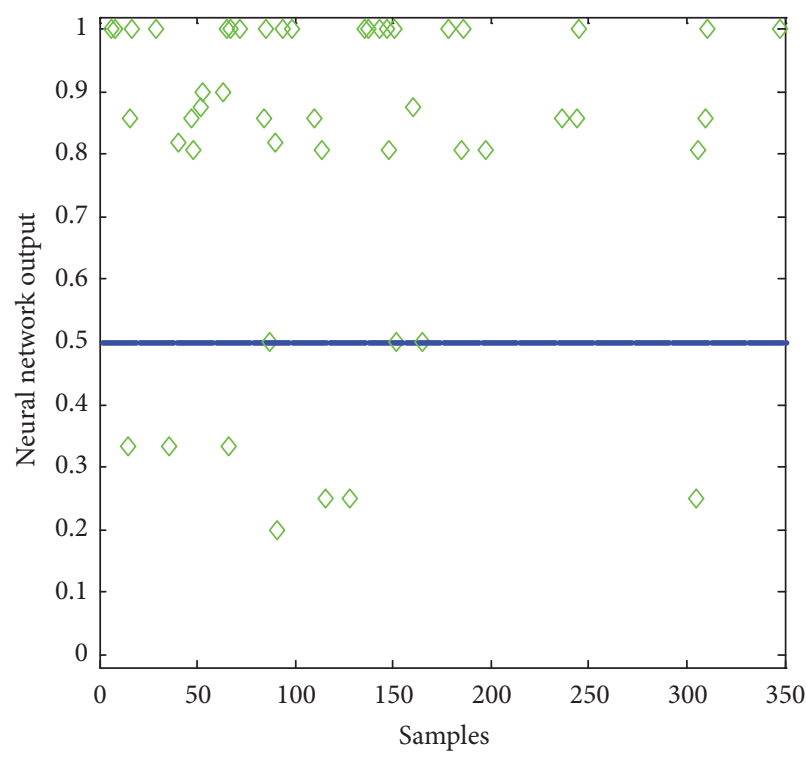

FIGURE 4: The RBF NN model classification result (accuracy: 81.6\%).

network training results with the real acquisition results, it could be observed from Figures 3 and 4 that the model training accuracy has achieved $97.4 \%$, whereas the validation classification result is $81.6 \%$. It should be noted that the less accuracy of the validation is mainly due to the fact that the validation data resource is only from the acquired spin-outs which may not have fully representing behaviors for all spinouts. The weight value vector has been obtained by means of the set-membership approach proposed in Section 3.3. With the trained weight value, the average value of the NN output is computed and equals 0.14 . Then, we may wonder how the following six factors (equity distributions) contribute to the acquisition of USOs. Thus, we modify the original data sets by increasing proportion of one of the factors and decreasing the ratio of other factors correspondingly. Then, the average value 
TABLE 1: Contribution results of different factors.

\begin{tabular}{lccc}
\hline Factor & $\begin{array}{c}\text { Change of } \\
\text { average value }\end{array}$ & $\begin{array}{c}\text { Positive or } \\
\text { negative }\end{array}$ & $\begin{array}{c}\text { Strong, } \\
\text { medium, or } \\
\text { weak }\end{array}$ \\
\hline Founders & +0.0448 & Positive & Weak \\
University & -0.0612 & Negative & Medium \\
Banks & +0.1228 & Positive & Strong \\
Business angels & +0.0406 & Positive & Weak \\
Venture capitals & +0.0813 & Positive & Medium \\
Other & +0.1193 & Positive & Strong \\
\hline
\end{tabular}

is computed by testing the modified data sets with the same weight value. If the average value increases, we believe that this factor contributes to the acquisition; otherwise this factor will not contribute to the acquisition or even discourage the acquisition. Meanwhile, the average value increases more meaning that the corresponding factor contributes to the acquisition more. The results are as shown in Table 1.

From the simulation results, it can be found that the increase of founders' equity has a positive effect on the acquisition of USOs, though the effect is weak; the increase of university's equity has a negative effect on the acquisition of USOs, and the effect is medium; the increase of banks' equity has a strong positive effect on the acquisition of USOs by the value as +0.1228 ; the increase of business angels' equity has a relatively weak positive effect on the acquisition of USOs, with the change value as +0.0406 ; the increase of venture capitals' equity has a positive effect on the acquisition of USOs, and the effect is medium; the increase of other equity has a positive effect on the acquisition of USOs, and the effect is strong.

It is demonstrated that the increases of founders, financial providers (including banks, business angels, and venture capitals), and other equity are conducive to the acquisition of USOs, and banks' equity is the most influential factor for the acquisition. The main reason for the results is possibly due to the fact that founders and financial are more concerned about the benefits and profits of USOs; therefore, the increase of their equities is inclined to the acquisition. The reason why other equity has a positively strong effect on the acquisition might likely be that this is captured by other businesses, who may be forming strategic joint ventures or alliances with the USOs. The effect of business angels' equity is much less, and probably the reason for this is that business angels come from diverse backgrounds ranging from former entrepreneurs to finance specialists, all of whom are inclined to target the less risky proposals compared to those favored by venture capitalists. On the other hand, the increase of university equity or other equity is not conducive to be acquired for USOs, and it could be explained that universities are more concerned about the emergence and transfer of knowledge and technology compared to the economic benefits.

\section{Conclusions and Suggestions}

This paper adopts RBF NN model to investigate the impacts of six equities (including founders, university, banks, business angels, venture capitals, and others) on the acquisition of USOs. Based on the sample data of 350 UK USOs, the average distribution of spin-out equity at formation is first calculated. According to this distribution, an RBF NN model is employed to forecast the effects of each equity change on the acquisition. To improve the classification accuracy, the novel set-membership method is adopted in the training process of the RBF NN. Furthermore, a simulation test is carried out to test the effects of six equities on the acquisition of USOs. The simulation results show that the increase of university's equity has a negative effect on the acquisition of USOs; however, the increase of remaining five equities has positive impact. The results demonstrate that the increases of founders, financial providers (including banks, business angels, and venture capitals), and other equity are conducive to the acquisition of USOs.

According to the key findings of this paper, some policy implications are proposed as references for decision-making by policymakers, founders, universities, financing providers, and so on.

(1) If USOs would like to be acquired in the future, a reasonable equity allocation should be ensured by the time of USOs formation when creating businesses from university.

(2) If the equity distribution was determined when the business was formed, and the USO has not been acquired all the time, they should adjust equity distribution and increase the percentage of founders' equity, financial providers' equity, or other equity appropriately.

(3) In order to be acquired, USOs should encourage and explore multiple investments and financing channels.

\section{Conflicts of Interest}

The authors declare that they have no conflicts of interest.

\section{Acknowledgments}

This research is financially supported by the National Natural Science Foundation of China under Grants 71302028, 71472057, and 71602041, Heilongjiang Province Natural Science Fund (LC2017031), and Fundamental Scientific Research Funds for the Central Universities (HEUCF170903, HEUCFW170908, and HEUCFW170904).

\section{References}

[1] R. T. Harrison and C. Leitch, "Voodoo institution or entrepreneurial university? spin-off companies, the entrepreneurial system and regional development in the UK," Regional Studies, vol. 44, no. 9, pp. 1241-1262, 2010.

[2] S. J. Franklin, M. Wright, and A. Lockett, "Academic and surrogate entrepreneurs in university spin-out companies," The Journal of Technology Transfer, vol. 26, no. 1-2, pp. 127-141, 2001.

[3] D. S. Siegel, D. Waldman, and A. Link, "Assessing the impact of organizational practices on the relative productivity of 
university technology transfer offices: an exploratory study," Research Policy, vol. 32, no. 1, pp. 27-48, 2003.

[4] S. Hess and R. Y. Siegwart, "University technology incubator: technology transfer of early stage technologies in crossborder collaboration with industry," Business and Management Research, vol. 2, no. 2, pp. 22-36, 2013.

[5] C. L. Lin, J. F. Wang, C. Y. Chen, C. W. Chen, and C. W. Yen, "Improving the generalization performance of RBF neural networks using a linear regression technique," Expert Systems with Applications, vol. 36, no. 10, pp. 12049-12053, 2009.

[6] H. K. K. Tung and M. C. S. Wong, "On the formulation of credit barrier model using radial basis functions," Journal of the Operational Research Society, vol. 65, no. 9, pp. 1437-1452, 2014.

[7] A. Guarin, X. Liu, and W. L. Ng, "Enhancing credit default swap valuation with meshfree methods," European Journal of Operational Research, vol. 214, no. 3, pp. 805-813, 2011.

[8] P. A. Gutiérrez, M. J. Segovia-Vargas, S. Salcedo-Sanz et al., "Hybridizing logistic regression with product unit and RBF networks for accurate detection and prediction of banking crises," Omega , vol. 38, no. 5, pp. 333-344, 2010.

[9] A. Lockett and M. Wright, "Resources, capabilities, risk capital and the creation of university spin-out companies," Research Policy, vol. 34, no. 7, pp. 1043-1057, 2005.

[10] P. Mustar, M. Renault, M. G. Colombo et al., "Conceptualising the heterogeneity of research-based spin-offs: a multidimensional taxonomy," Research Policy, vol. 35, no. 2, pp. 289308, 2006.

[11] A. Walter, M. Auer, and T. Ritter, "The impact of network capabilities and entrepreneurial orientation on university spinoff performance," Journal of Business Venturing, vol. 21, no. 4, pp. 541-567, 2006.

[12] M. J. Benner and M. L. Tushman, "Exploitation, exploration, and process management: the productivity dilemma revisited," Academy of Management Review (AMR), vol. 28, no. 2, pp. 238256, 2003.

[13] M. Steffensen, E. M. Rogers, and K. Speakman, "Spin-offs from research centers at a research university," Journal of Business Venturing, vol. 15, no. 1, pp. 93-111, 2000.

[14] M. Klofsten and D. Jones-Evans, "Comparing academic entrepreneurship in europe-The case of sweden and ireland," Small Business Economics, vol. 14, no. 4, pp. 299-309, 2000.

[15] R. De Coster and C. Butler, "Assessment of proposals for new technology ventures in the UK: characteristics of university spin-off companies," Technovation, vol. 25, no. 5, pp. 535-543, 2005.

[16] R. Landry, N. Amara, and M. Saílhi, "Patenting and spinoff creation by Canadian researchers in engineering and life sciences," The Journal of Technology Transfer, vol. 32, no. 3, pp. 217-249, 2007.

[17] E. B. Roberts, Entrepreneurs in High Technology, Oxford University Press, New York, NY, USA, 1991.

[18] European Commission, Progress Report on the Risk Capital Action Plan, Brussels and Luxembourg, 2000a.

[19] European Commission, Innovation in a Knowledge Driven Economy, Brussels and Luxembourg, 2000b.

[20] A. Muscio, D. Quaglione, and L. Ramaciotti, "The effects of university rules on spinoff creation: the case of academia in Italy," Research Policy, vol. 45, no. 7, pp. 1386-1396, 2016.

[21] J. De Jesus Rubio, P. Angelov, and J. Pacheco, "Uniformly stable backpropagation algorithm to train a feedforward neural network," IEEE Transactions on Neural Networks and Learning Systems, vol. 22, no. 3, pp. 356-366, 2011.
[22] O. Abedinia and N. Amjady, "Net demand prediction for power systems by a new neural network-based forecasting engine," Complexity, vol. 21, no. S2, pp. 296-308, 2016.

[23] M.-C. Pai, "RBF-based discrete sliding mode control for robust tracking of uncertain time-delay systems with input nonlinearity," Complexity, vol. 21, no. 6, pp. 194-201, 2016.

[24] L. V. Ballestra and G. Pacelli, "Pricing European and American options with two stochastic factors: a highly efficient radial basis function approach," Journal of Economic Dynamics and Control, vol. 37, no. 6, pp. 1142-1167, 2013.

[25] R. T. L. Chan and S. Hubbert, "Options pricing under the one-dimensional jump-diffusion model using the radial basis function interpolation scheme," Review of Derivatives Research, vol. 17, no. 2, pp. 161-189, 2014.

[26] M. Thompson, "Counterparty credit risk pricing and measurement of swaption portfolios," The Journal of Computational Finance, vol. 18, no. 1, pp. 31-64, 2014.

[27] H. I. Erdal and A. Ekinci, "A comparison of various artificial intelligence methods in the prediction of bank failures," Computational Economics, vol. 42, no. 2, pp. 199-215, 2013.

[28] M. Thompson, "Optimal economic dispatch and risk management of thermal power plants in deregulated markets," Operations Research, vol. 61, no. 4, pp. 791-809, 2013.

[29] C. Wu and D. Barnes, "A dynamic feedback model for partner selection in agile supply chains," International Journal of Operations and Production Management, vol. 32, no. 1, pp. 79-103, 2012.

[30] G. Kapetanios and A. P. Blake, "Tests of the martingale difference hypothesis using boosting and RBF neural network approximations," Econometric Theory, vol. 26, no. 5, pp. 13631397, 2010.

[31] http://www.spinoutsuk.co.uk/listings/company-listings/.

[32] D. Chen and W. Han, "Prediction of multivariate chaotic time series via radial basis function neural network," Complexity, vol. 18 , no. 4, pp. 55-66, 2013.

[33] N. Q. Huy, D. S. Kim, and N. V. Tuyen, "Existence theorems in vector optimization with generalized ORDer," Journal of Optimization Theory and Applications, vol. 174, no. 3, pp. 728745, 2017.

[34] W. Yu and J. D. J. Rubio, "Recurrent neural networks training with stable bounding ellipsoid algorithm," IEEE Transactions on Neural Networks and Learning Systems, vol. 20, no. 6, pp. 983991, 2009.

[35] D. J. R. Jose, Y. Wen, and F. Andres, "Neural network training with optimal bounded ellipsoid algorithm," Neural Computing and Applications, vol. 18, pp. 623-631, 2009. 


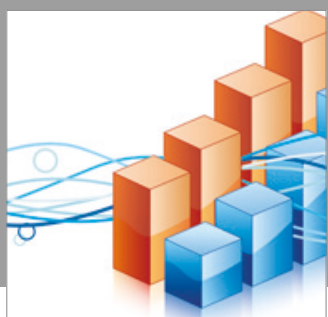

Advances in

Operations Research

vatersals

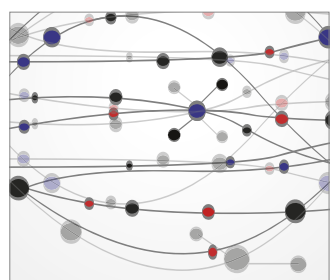

\section{The Scientific} World Journal
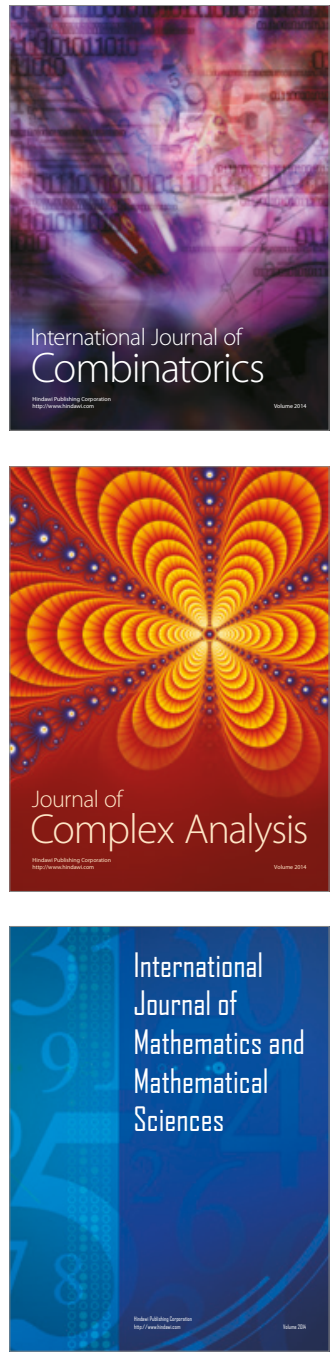
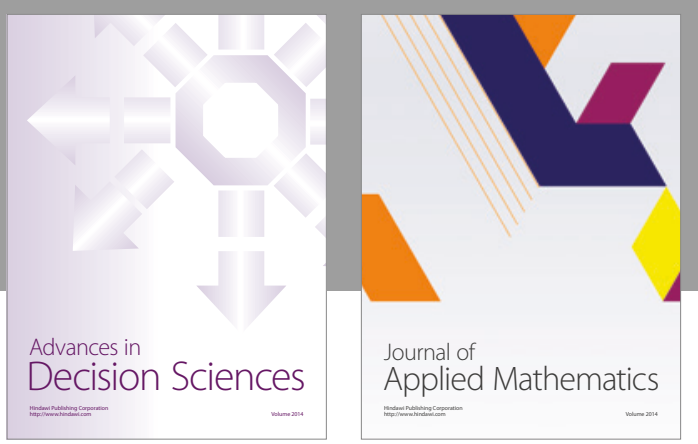

Algebra

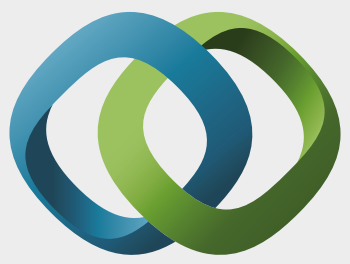

\section{Hindawi}

Submit your manuscripts at

https://www.hindawi.com
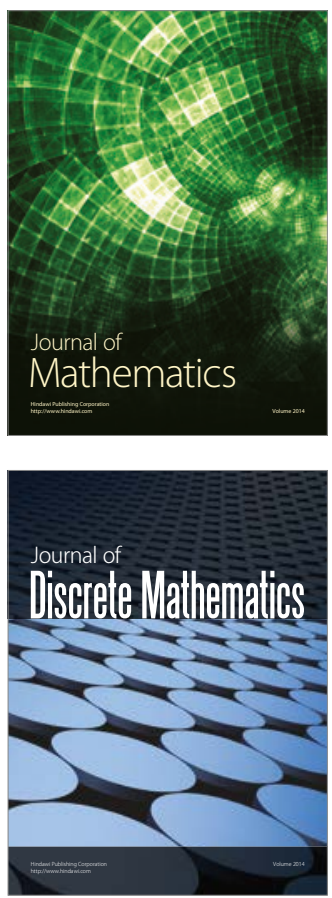

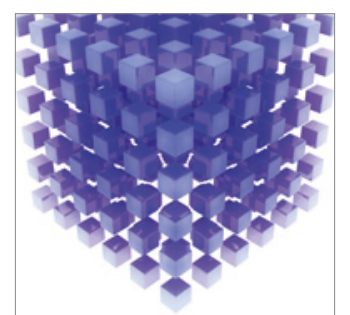

Mathematical Problems in Engineering
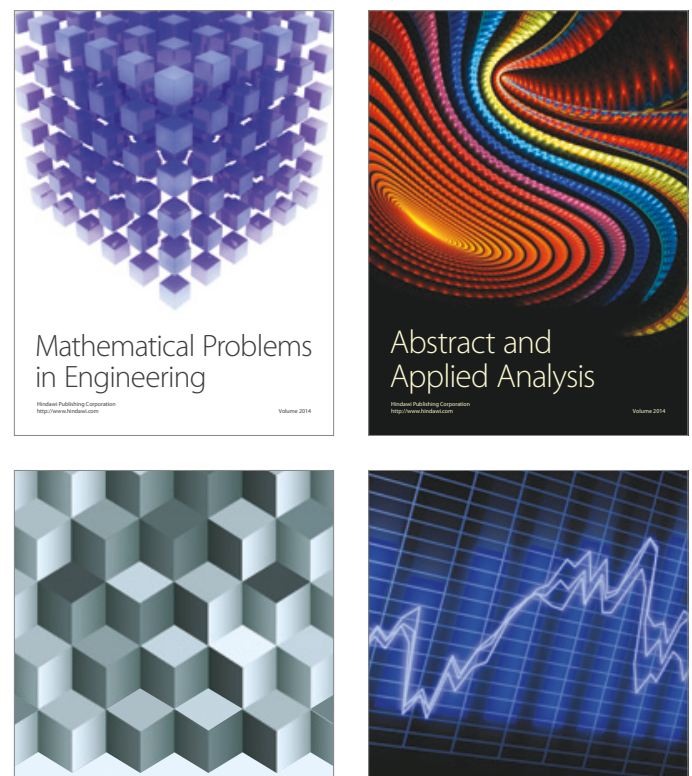

Journal of

Function Spaces

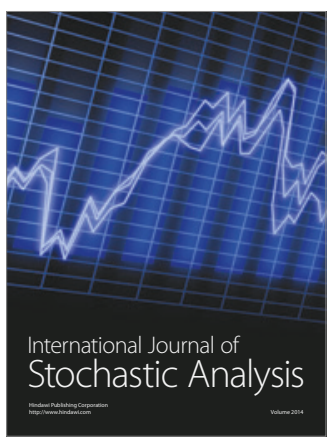

Probability and Statistics
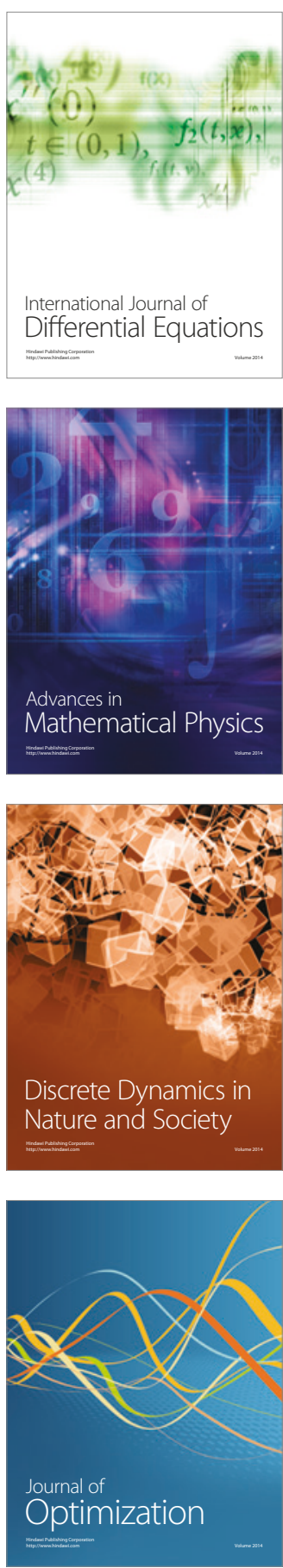